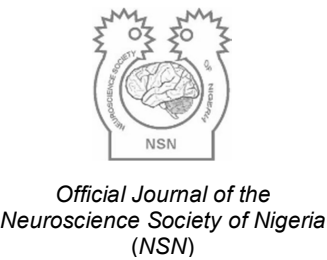

ORIGINAL ARTICLE

http://doi.org/10.47081/njn2021.12.2/004

ISSN 1116-4182

\title{
Neurocognitive and Neuroarchitectural Changes in the Prefrontal Cortex of Wistar Rats Treated with Highly Active Antiretroviral Therapy
}

\author{
Olufunke Dosumu ${ }^{1}$, Edidiong Akang ${ }^{1}$, Edem Edem ${ }^{2}$, \\ Samuel Afolayan ${ }^{1}$, Alani S. Akanmu ${ }^{3}$ \\ ${ }^{1}$ Department of Anatomy, College of Medicine, University of Lagos, Lagos, Nigeria. \\ ${ }^{2}$ Department of Anatomy, College Medicine and Health Sciences, Afe Babalola University, Ado-Ekiti, Nigeria. \\ ${ }^{3}$ Department of Haematology and Blood Transfusion, College of Medicine, University of Lagos, Lagos, Nigeria
}

Received:

March 2021

Accepted: August 2021

\begin{abstract}
The use of highly active antiretroviral therapy has proven to be highly effective in the treatment of human immunodeficiency virus 1 (HIV-1) infection. However, its impact on cognition has not been fully explored. This study was designed to assess the impacts of antiretroviral therapy on cognitive function and histoarchitecture of the prefrontal cortex of Wistar rats. Forty adult male Wistar rats weighing 180$200 \mathrm{~g}$ were randomly assigned to 4 groups: control, tenofovir, lamivudine and efavirenz $(n=10)$, which received $1 \mathrm{ml}$ distilled water and $6 \mathrm{mg} / \mathrm{kg}, 6 \mathrm{mg} / \mathrm{kg}$ and $12 \mathrm{mg} / \mathrm{kg}$, respectively. Spatial memory scores were assessed using the $\mathrm{Y}$-maze test. Following behavioural studies, the animals were euthanized, and their whole brains harvested. The prefrontal cortex was sectioned and processed for oxidative stress, histological and immunohistochemical analyses. There was a significant decrease in percentage alternation evaluated from the right/wrong decisions scored from the tenofovir and lamivudine groups, compared to the control group $(p<0.05)$. malondialdehyde (MDA) and reduced glutathione $(\mathrm{GSH})$ levels were elevated following lamivudine and tenofovir exposure in the rats' prefrontal cortices, respectively, compared to control $(p<0.05)$. There were also significant alterations of cortical pyramidal cells in the tenofovir and lamivudine groups. Additionally, marked astrogliosis with increased glial fibrillary acidic protein expression was observed, consistent with the structural alterations, especially in the lamivudine group. Our findings suggest that, of the three highly active antiretroviral therapy (HAART) drugs studied, lamivudine may be a major culprit in the progressive neurological damage and cognitive impairment in HIV-infected individuals on HAART.
\end{abstract}

Key words: Antiretroviral therapy; Tenofovir; Lamivudine; Efavirenz; Cognition; Prefrontal cortex

\section{INTRODUCTION}

Significant progress has been made in mitigating and preventing human immunodeficiency virus (HIV)related complications, including HIV-associated dementia. Antiretroviral medicines work by preventing the multiplication of human immunodeficiency virus (HIV), reducing its virulence (De Clercq 2009; Broder 2010).

Current international treatment guidelines for HIV entails the use of three antiretroviral drugs to attain effective suppression of HIV-1 RNA replication (WHO 2021). Of the three antiretroviral drugs recommended, two are from the nucleoside reverse transcriptase inhibitors (NRTI) family, usually, tenofovir disoproxil fumarate and lamivudine, while the third is from nonnucleoside reverse transcriptase inhibitors (NNRTIs)

Correspondence: Edidiong Akang, $\mathrm{PhD}$, Department of Anatomy, College of Medicine, University of Lagos, P.M.B. 12003, Idi-Araba, Lagos, Nigeria. E-mail: enakang@cmul.edu. ng; Phone: +2348065299278 
usually either efavirenz, integrase strand transfer or a protease inhibitor (Soriano et al. 2017). NRTIs cling to the HIV-1 reverse transcriptase and serve as chain terminators preventing the completion of pro-viral DNA synthesis. NNRTIs also inhibits reverse transcriptase-dependent DNA synthesis (Das and Arnold 2013).

Highly active antiretroviral therapy (HAART) has remarkably improved the quality of life of people living with HIV but there remain about $20-50 \%$ of people living with HIV (PLHV) who report different neurological disorders (Chen et al. 2013; Gabbai et al. 2013; Heaton et al. 2015). In the pre- antiretroviral therapy era, HIV-associated neurocognitive disorders (HAND) affected as high as $50 \%$ of PLHV (Grant et al. 1988; Saylor et al. 2016). However, the sheer severity of HAND has been reduced with only $2-3 \%$ of PLHV being afflicted with HIV-associated dementia. Most HAND cases are diagnosed with asymptomatic neurocognitive impairments or minor neurocognitive disorders (Saylor et al. 2016). It is quite confounding to observe from reports a high prevalence of some categories of HAND since people with HIV (PWH) on antiretroviral therapy often have low to undetectable viral loads. Studies have shown that low-level HIV protein expression may persist in HIV+ patients even on antiretroviral therapy (Ganor et al. 2019; Prevedel et al. 2019), but mounting evidence suggests that antiretroviral medications may also underlie some of the neurological dysfunctions observed (Robertson et al. 2012; Lorber 2013; $\mathrm{Xu}$ et al. 2017; Llibre et al. 2018; Soontornniyomkij et al. 2018). In fact, studies have implicated Chronic activation of astrocytes in HIVassociated neurocognitive disorder (HAND) and in the development of neurodegenerative diseases including Alzheimer's disease (AD) (GuillamónVivancos et al. 2015; Ru and Tang 2017). Astrocyte reactivity or astrogliosis refers to a series of molecular, morphological and functional changes astrocytes undergo in response to damage or injury to the central nervous system (CNS).

In our previous study, it was observed that the combination of highly active antiretroviral drugs comprising tenofovir, efavirenz and lamivudine increased oxidative stress, adversely affected memory and induced degeneration of neurons (Akang et al. 2019). Hence, this study was aimed at investigating the effects of tenofovir, efavirenz and lamivudine on cognitive function and histoarchitecture of the prefrontal cortex of Wistar rats

\section{MATERIALS AND METHODS}

\section{Chemical and Reagents}

The antiretroviral drugs including; tenofovir, efavirenz and lamivudine tablets of Merck Pharmaceutical Company, USA, were obtained from Chevron Clinic,
Gbagada and AIDS Prevention Initiative in Nigeria (APIN) Centre of Lagos University Teaching Hospital.

\section{Experimental Animals}

Forty adult male Wistar rats weighing 180-200 g were randomly assigned to four different groups $(n=10)$ : control, tenofovir, lamivudine, and efavirenz. They were allowed to acclimatise for two weeks in the Animal house of the Department of Anatomy, College of Medicine, University of Lagos. They were exposed to $12 \mathrm{~h}$ light-dark cycle and allowed access to food and water ad libitum. The College of Medicine, University of Lagos' Health Research Ethics Committee approved this study with protocol number CMUL/HREC/03/17/113, and were conducted in accordance with the National Institute of Health Guide for the Care and Use of Laboratory Animals (NIH Publications No. 80-23) revised 1996.

\section{Treatment}

The animals were allowed to acclimatise for two weeks, weighed and randomly assigned to four different groups $(n=10)$ : control, tenofovir, lamivudine and efavirenz; and treated once daily for 42 consecutive days via oral administration. Treatments were as follows: control $(0.5 \mathrm{ml}$ distilled water $), 6$ $\mathrm{mg} / \mathrm{kg}$ body weight (b.w.) of tenofovir, $6 \mathrm{mg} / \mathrm{kg} \mathrm{b.w.} \mathrm{of}$ lamivudine and $12 \mathrm{mg} / \mathrm{kg}$ b.w. of efavirenz (Tibalinda et al. 2016).

\section{Behavioural Assessment Y-Maze Test}

Pre- and post-treatment spatial memory scores were assessed a week before and on the 42nd day of experiment respectively using the $\mathrm{Y}$-maze test as previously reported (Ademola et al. 2016) in the Behavioural Testing Room of the Department of Anatomy, College of Medicine of the University of Lagos. Briefly, rats were placed at the junction of the three arms of the $Y$ maze and allowed to make their arm decisions for a total test duration of $5 \mathrm{~min}$. The frequencies of correct decisions/alterations ( $A B C$, $A C B, B C A, B A C, C B A$ or $C A B$ ) between the arms $(A B C)$ were recorded to determine the memory index (percentage of correct alteration). The maze was cleaned with $70 \%$ ethanol between the trials to eliminate olfactory cues. The percentage alternation was calculated thus:

$$
\% \text { Alternation }=\frac{\text { No. of right decisions }}{\text { No. of total arm entries }-2} \times 100
$$

\section{Biochemical Analyses of Oxidative Stress Markers}

Dissected prefrontal cortices of five animals per group were homogenised and centrifuged at $4,000 \mathrm{~g}$ for $5 \mathrm{~min}$ to obtain supernatant for the measurement of malondialdehyde (MDA), reduced glutathione $(\mathrm{GSH})$, superoxide dismutase (SOD) and catalase 


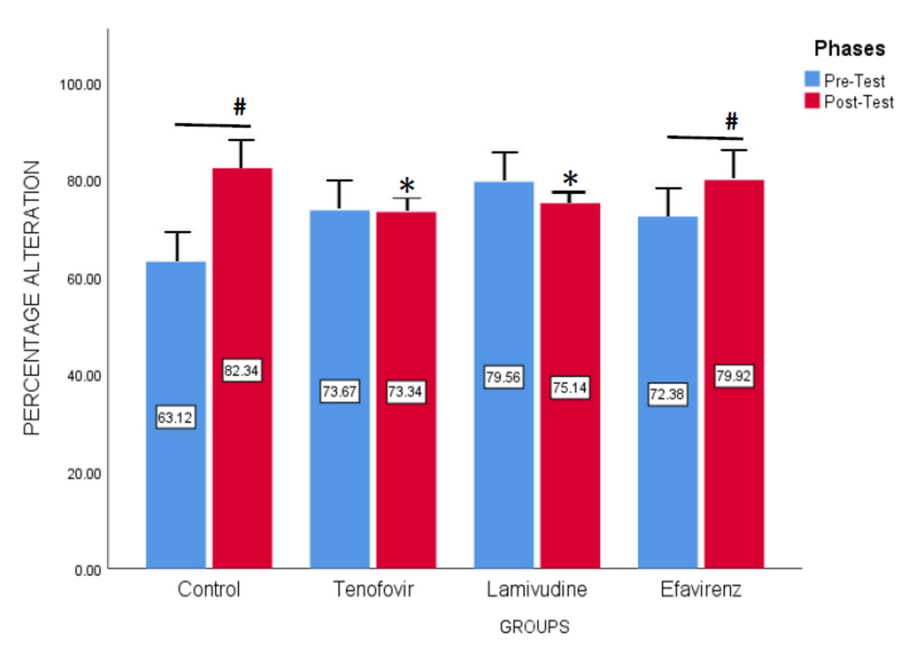

Fig. 1: Percentage alternation following pre and posttreatment $\mathrm{Y}$-maze assessment of lamivudine, efavirenz and tenofovir-treated Wistar rats. \# $p<0.05$ between pre and post treatments while * $p<0.05$ compared to control.

(CAT) levels. The supernatant and $60 \mu \mathrm{L}$ of $50 \mathrm{mM}$ sodium phosphate buffer ( $\mathrm{pH} 8.0)$ were incubated at $37^{\circ} \mathrm{C}$ for $15 \mathrm{~min}$, and then added to $70 \mu \mathrm{L}$ Ellman's reaction mixture in a $50 \mathrm{mM}$ sodium phosphate buffer to test AChE activity. Absorbance was measured at $405 \mathrm{~nm}$ after incubation at $37^{\circ} \mathrm{C}$.

\section{Measurement of Lipid Peroxidation}

Lipid peroxidation was measured by the reaction of MDA with thiobarbituric acid at $535 \mathrm{~nm}$ (Albro et al. 1986) using the extinction coefficient of MDA $156 / \mathrm{mM} / \mathrm{cm}$. The results were expressed as nmol/mg protein.

\section{Measurement of Glutathione Level}

Reduction in GSH was done using Ellman's method (Ellman 1959) modified by Hissin and Hilf (1976). The procedure entails the reduction of Ellman's reagent by -SH groups of GSH to form 2nitro-s mercaptobenzoic acid. The yellow colour was read immediately at $405 \mathrm{~nm}$. At standard GSH (0.001-0.1 mM), a calibrated curve was conducted and GSH concentrations were estimated as $\mathrm{nmol} / \mathrm{mg}$ protein.

\section{Measurement of Total Superoxide Dismutase Level}

Superoxide dismutase (SOD) activity was assayed based on the production of superoxide radicals in xanthine and xanthine oxidase reactions, which also reacts with 2-(4-iodophenyl)-3-(4-nitrophenol)5-phenyltetrazolium chloride (INT) to form a red formazan dye. The degree of inhibition measures the activity of SOD. A unit of SOD causes $50 \%$ inhibition of the rate of reduction of INT under the conditions of the assay. The assay protein concentrations of samples were adjusted to enable reaction linearity in accordance with manufacturer's instructions. The final SOD activity was expressed as units per gram of total proteins (IU/mg protein) (Grankvist et al. 1979).

\section{Measurement of Catalase Level}

Aliquots of supernatants from the prefrontal cortex were used to determine catalase levels by the addition of $500 \mu \mathrm{L}$ of $\mathrm{TiOSO}_{4}$ to the reaction tube. Catalase activity was assayed spectrophotometrically by measuring absorbance at $420 \mathrm{~nm}$ (Feuers et al. 1997).

\section{Histological/Immunohistochemical Analyses}
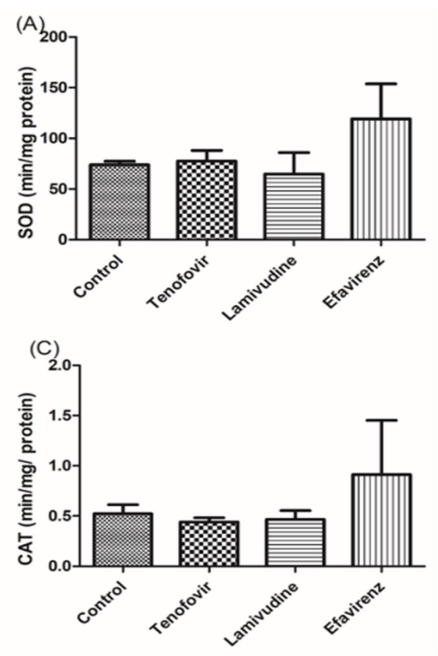
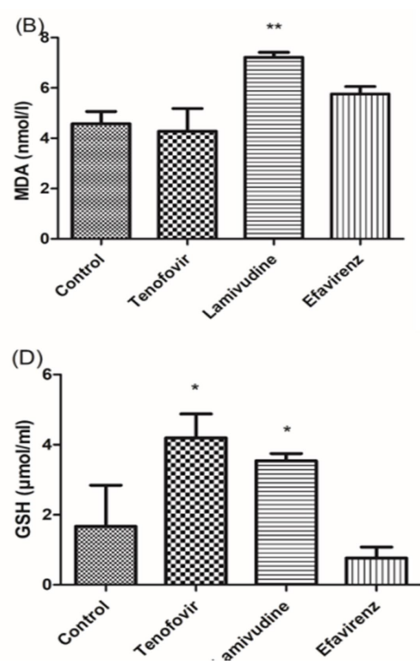

Fig. 2: Effect of lamivudine, tenofovir and efavirenz on SOD, MDA, CAT and GSH levels in the prefrontal cortex of Wistar rats. ${ }^{*} p<0.05$, ${ }^{* *} p<0.01$, significantly different from control.
For tissue collection and processing, the rats were euthanized with an intraperitoneal injection of ketamine hydrochloride (10 $\mathrm{mg} / \mathrm{kg}$ ) and xylazine (8 $\mathrm{mg} / \mathrm{kg}$ ). Five of the rats/group were perfused intracardially with normal saline and $10 \%$ formal saline for pre-fixation of the tissues. The whole brain was harvested, the pre-frontal cortex sectioned and post-fixed in $10 \%$ formal saline for tissue processing for paraffin wax embedding in accordance with Akang et al. (2019). Haematoxylin and eosin (H \& E) staining was done as described by Cardiff et al. (2014). The photomicrographs were captured and examined using the Leica DM 750 microscope with ICC 50 HD camera. 

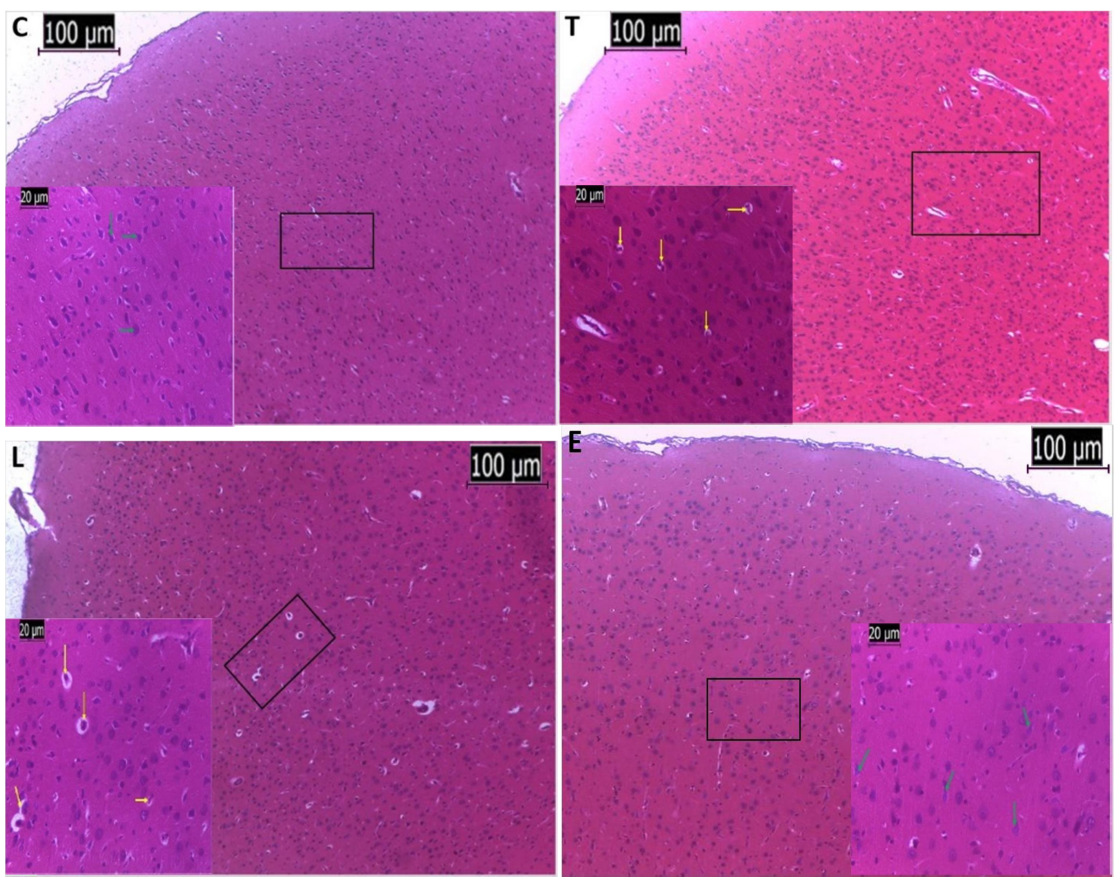

Fig. 3: Representative photomicrographs of the prefrontal cortex of Wistar rats of the control (C) and efavirenz (E) groups showing normal cortical neurons (green arrows). Tenofovir (T) and lamivudine (L) groups shows several pyknotic neurons (yellow arrows) $\times 100, \times 400$. Scale: $100 \mu \mathrm{m}, 20 \mu \mathrm{m}$.

For immunohistochemical analysis, deparaffinised sections of the prefrontal cortex were washed in 2 changes of $0.3 \% \mathrm{H}_{2} \mathrm{O}_{2}$ in methanol to stop the
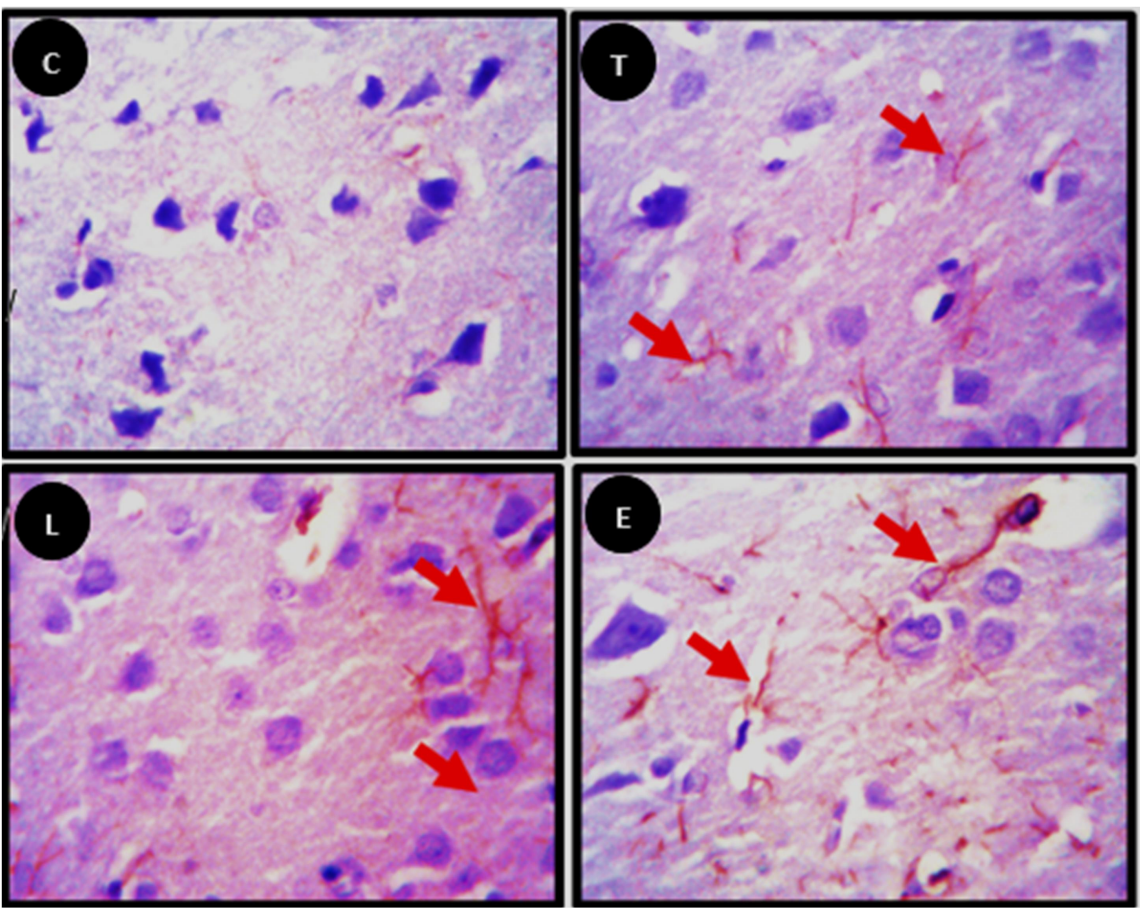

Fig. 4: Representative photomicrograph showing GFAP expression (red arrows) in the prefrontal cortex of Wistar rats. Control (C) group showing low reactivity to GFAP. Tenofovir (T), lamivudine (L) and efavirenz (E) showed high expression of GFAP. $\times 400$ endogenous peroxidase activity. Then, the sections were washed with $0.1 \mathrm{M}$ PBS (pH 7.4) with $0.3 \%$ Triton $\mathrm{X}-100$ and $5 \%$ foetal bovine serum. The sections were incubated overnight at $4^{\circ} \mathrm{C}$ with primary polyclonal antibody rabbit anti-GFAP 1:200 (Elabscience, E-AB-70040), followed by washes with $0.1 \mathrm{M}$ PBS, before being incubated for 90 min with mouse anti-rabbit IgG biotin conjugate 1:300 (Elabscience, E-AB-1030). Sections were washed in 0.1 M PBS, and binding sites of antibodies were revealed with 2-step plus poly-HRP anti mouse/rabbit IgG detection system with 3,31diaminobenzidine (DAB) soluteion (Elabscience, E-IR-R217). Sections were counterstained in haematoxylin (Akang et al. 2019).

\section{Statistical Analysis}

Paired t-test was used to compare between pre- and post- treatment in the Y-maze neurobehavioral test; while one-way ANOVA was used to compare changes between post treatment and control groups following behavioural assessment. Data were expressed as mean \pm S.E.M. Statistical significance of differences between means was determined using one-way ANOVA via the IBM SPSS 20. P-values less than 0.05 were considered significant using Tukey`s post hoc test.

\section{RESULTS}

\section{Y-maze Test}

The Y-maze memory performance assessment result showed a significant increase in percentage alternation evaluated from the right/wrong decisions scored in the pre- and posttreatment of control and efavirenz groups but this was not observed in other groups. There was also a significant decrease in tenofovir and lamivudine groups compared to the control group $(p<0.05$, Fig. $1)$. 


\section{Assessment of Oxidative Stress Markers in the Prefrontal Cortex}

The MDA levels of lamivudine group increased significantly $(p<0.05)$ compared to the control (Fig. 2B). There was significant increased GSH levels $(p<0.01)$ in the tenofovir and lamivudine groups when compared to the control (Fig. 2D). There was no significant difference in SOD and CAT levels between the control and the treated groups (Fig. 2A and 2C).

\section{Histological Analysis}

The $H$ \& $E$ staining technique revealed that the neuronal pyramidal cells appeared unaffected and evenly distributed in the multiform layer of the prefrontal cortex of the control group. The prefrontal cortex sections from the tenofovir and lamivudinetreated rats revealed depletion, vacuolation and degeneration of pyramidal neurons in the multiform layer. The histomorphology of the prefrontal cortex of the efavirenz-treated rats showed mostly unaffected histomorphology (Fig. 3).

\section{Immunohistochemical Analysis}

The immunohistochemical demonstration revealed increase expression of GFAP and activation of astrocytes in the prefrontal cortex of the HAART (tenofovir, lamivudine and efavirenz) exposed groups when compared to the control group (Fig. 4).

\section{DISCUSSION}

Therapy with three medications has been the standard for patients with HIV. Despite the reduction in the mortality rate of HIV infected patients through the introduction of HAART, the occurrence of HIVassociated neurocognitive disorders (HAND) persists in patients. In our previous study (Akang et al. 2019), there was marked neurotoxicity from the combined therapy of tenofovir (NRTI), lamivudine (NRTI), and efavirenz (NNRTI) on cognition. In the present study, we sought to identify the potential effects associated with individual drugs in the HAART combined therapy.

Cognitive assessment in this study is in agreement with earlier studies suggesting that HAART may be responsible for the cognitive deficits observed (Robertson et al. 2007; Zulu et al. 2018; Fields et al. 2019). The Y-maze performance test following treatment with lamivudine and tenofovir showed a decrease in percentage alternation evaluated from the right/wrong decisions scored in the tenofovir and lamivudine groups, compared to the control group which indicates a negative impact on learning and memory. It is also supported by the degeneration of neurons observed in the histological results of these groups. However, a significant increase in percentage alternation was seen in the efavirenztreated rats compared to the control, demonstrating a potential memory enhancing benefit of efavirenz. Recent studies have shown that efavirenz has the potential to upregulate cytochrome $P_{450} \quad 46 \mathrm{~A} 1$ (CYP46A1) activity. CYP46A1 is expressed in many neurons and regulates the production of 24hydroxycholesterol which is a modulator of $\mathrm{N}$-methylD-aspartate receptors (NMDAR) involved in memory and cognition (Maioli et al. 2013; Petrov et al. 2019).

A nexus has been established between oxidative stress and neurodegenerative diseases pathogenesis, including HAND (Shibata and Kobayashi 2008; Schifitto et al. 2009). Oxidative stress due to excessive reactive oxygen species (ROS) production disrupts reduction-oxidation balance and reduces protein folding, which will subsequently impair cellular metabolism (Pejman et al. 2020). We examined the effect of HAART medications (tenofovir, lamivudine and efavirenz) on antioxidative status by determining the activities of SOD, MDA, GSH and CAT in the prefrontal cortices of rats exposed to these drugs. SOD is the antioxidant enzyme responsible for the dismutation of free radicals generated during HAART (tenofovir and lamivudine) metabolism to hydrogen peroxide, which is then changed to water by catalase and glutathione (Sen et al. 2010). Pronounced oxidative stress following lamivudine administration was observed in the present study as demonstrated by the increased ROS production and lipid peroxidation in the prefrontal cortex. Our study agrees with Guo et al. (2013), who reported evidence of antiretroviral therapy-induced oxidative stress in neurons associated with impaired mitochondrial function. Indeed, oxidative stress and mitochondrial dysfunction has been fingered as a key contributor to the pathogenesis of many neurocognitive disorders. Even though it might be premature to explain the exact mechanism responsible for the higher levels of GSH observed in the treatment groups, in contrast to the high MDA levels, it may possibly be as a result of the release of GSH into the extracellular microenvironment by astrocytes which then generates precursors for neuronal GSH synthesis (Hirrlinger et al. 2002).

Results from the present study showed that the lamivudine and tenofovir might adversely alter the histoarchitectural integrity of the prefrontal cortex, possibly due to increased mitochondrial damage, which is a source of free radicals (lkekpeazu et al. 2020). It should be noted that the NNRTIs have also been implicated in the oxidative stress hypothesis. It has been shown to inhibit creatinine kinase and cytochrome c oxidase-mediated mitochondrial processes thereby enabling an environment for the production of reactive oxygen species that consequently drives oxidative stress (Funes et al. 2015; Hung et al. 2017).

However, in the present study, efavirenz did not alter the morphology of the prefrontal cortex. Tenofovir and lamivudine treated groups produced significant 
distortion of the cortical histomorphology. These results are in tandem with Ferrer and Rakhmanina (2013) who reported peripheral neuropathy in mice treated with tenofovir and the study of Peter et al. (2017) who reported shrunken Purkinje cells and distorted granular layer in the cerebellum of rats treated with lamivudine.

Several studies highlighting the detrimental effects that antiretroviral drugs have on neural structures are often through mechanisms involving oxidative stress. Whether these drugs also affect the factors involved in the processes of neuroinflammation and neuroplasticity is not clear at the moment. Our study hypothesises that the HAART (tenofovir, lamivudine and efavirenz) exposure may induce neuroinflammation with marked astrogliosis. Histologically, neuroinflammation is characterized by astrogliosis (Haim et al. 2015) and the production of pro-inflammatory mediators (Medeiros and LaFerla 2013). From this study, the antiretroviral drugs administered for six weeks markedly induced astrogliosis in the prefrontal cortices of the tenofovirand lamivudine-treated groups when compared to the control group. These results, therefore, suggest that both tenofovir and lamivudine can induce an inflammatory cascade in the brain, which drives neurodegeneration as seen in HAND (Rojo et al. 2008; Gelders et al. 2018). It is possible that in these conditions, reactive astrocytes or microglia may instigate the production of pro-inflammatory proteins associated with the onset of various neurodegenerative diseases (Lu et al. 2010; Vivithanaporn et al. 2016), including AIDS-induced parkinsonism (Devine et al. 2018). Our results agree with findings from a previous investigation that showed that administration of efavirenz increased astrogliosis in mice (Petrov et al. 2019). HAART-induced astrogliosis is possibly via the promotion of oxidative stress as seen in this study (Daverey and Agrawal 2016). Studies have shown that disruption of DNA synthesis in mitochondria in the presence of NRTIs is as a result of mitochondrial enzyme, DNA polymerase gamma impairment (Apostolova et al. 2010; Smith et al. 2017). The results of our study is therefore consistent with previous studies that demonstrated NRTIs, in this case, tenofovir and lamivudine induce oxidative damage and astrogliosis in the prefrontal cortex of rats (Tamagno et al. 2008; de Oliveira et al. 2014). This suggests that ART may induce memory impairment or further the risk of HIVassociated neurocognitive diseases.

Our findings indicating activation of astrocyte suggest that CNS-penetrating antiretroviral medications in neurologically active combined antiretroviral therapy (Neuro-CART) (Cysique et al. 2004; Letendre et al. 2008), may not confer significant protection on astrocytes against HIV infection. This agrees with a study by Gray et al. (2013), where they reported that NRTIs like lamivudine had insufficient HIV-1 inhibitory activity in astrocytes. In a general sense, the seeming higher neurotoxic capacity of lamivudine among the two NRTIs used in the present study compared to efavirenz could be due to the fact that, lamivudine as an NRTI requires activation from its latent form by different phosphorylation events, indicating that the drug is only effective against the HIV-1 reverse transcriptase once the drug is triphosphorylated; and alteration in these events can lead to differences in cellular uptake of NRTIs, inefficient or incomplete drug activation. This poor protection of NRTIs against HIV infection on astrocyte can compromise brain homeostasis fundamentally regulated by astrocytes, and can promote the development and progression of HAND in HIV-infected persons.

\section{Conclusion}

The emergence of HAART has enhanced the possibility of managing HIV-1 infection; nevertheless, the occurrence of chronic disease associated with neurocognitive disorders persists in $\mathrm{HIV}+$ patients. Several reports have unveiled the possibility that long-term exposure to HAART furthers the pathogenesis of HAND aside the HIV itself. Our findings therefore suggest that of the three HARRT drugs studied, lamivudine may be a major culprit in the progressive neurological damage and cognitive impairment in HIV-infected individuals on HAART.

\section{Grants and financial support Nil}

\section{Conflict of Interest}

None declared.

\section{Acknowledgement}

This study was supported by the AIDS Prevention Initiative of Nigeria (APIN), Lagos University Teaching Hospital, Lagos, Nigeria.

\section{Authors Contribution}

OD and EA: Conceptualization, visualization, methodology, validation, final draft of manuscript; SA: resources, investigation, data curation, project administration; EE and SA: Formal analysis and writing original draft. OD, EA and ASA: Supervision, resources, review and editing.

\section{REFERENCES}

Ademola, O., Edem, E., Olufunke, D. and Oladunni, K. (2016) Cognitive-enhancing and neurotherapeutic prospects of viscum album in experimental model of alzheimer's disease. African J Cell Pathol. 7:11-16. Akang, E.N., Dosumu, O.O., Afolayan, O.O., Fagoroye, A.M., Osiagwu, D.D., Usman, I.T., et al. (2019) Combination antiretroviral therapy (cART)induced hippocampal disorders: Highlights on 
therapeutic potential of naringenin and quercetin. IBRO Reports. 6:137-146. https://doi.org/10.1016/j. ibror.2019.04.002

Albro, P. W., Corbett, J.T. and Schroeder, J.L. (1986) Application of the thiobarbiturate assay to the measurement of lipid peroxidation products in microsomes. J Biochem Biophysical Methods. 13(3): 185-194. https://doi.org/10.1016/0165-022X(86)9009 $\underline{2-8}$

Apostolova, N., Gomez-Sucerquia, LJ., Moran, A., Alvarez, A., Blas-Garcia, A. and Esplugues, J. V. (2010) Enhanced oxidative stress and increased mitochondrial mass during efavirenz-induced apoptosis in human hepatic cells. $\mathrm{Br} \mathrm{J}$ Pharmacol. 160(8): 2069-2084. https://doi.org/10.1111/j.14765381.2010.00866.x

Ben Haim, L., Carrillo-de Sauvage, M.A., Ceyzériat, K. and Escartin, C. (2015) Elusive roles for reactive astrocytes in neurodegenerative diseases. Frontiers Cellular Neurosci. 9:278. https://doi.org/10.3389/ fncel.2015.00278

Broder, S. (2010) The development of antiretroviral therapy and its impact on the HIV-1/AIDS pandemic. Antiviral Res. 85(1):1-18. https://doi.org/10.1016/j. antiviral.2009.10.002

Cardiff, R.D., Miller, C.H. and Munn, R.J. (2014) Manual hematoxylin and eosin staining of mouse tissue sections. Cold Spring Harbor Protoc. 2014(6): 655-658. https://doi.org/10.1101/pdb.prot073411

Chen, H., Clifford, D.B., Deng, L., Wu, K., Lee, A.J., Bosch, R.J., et al. (2013) Peripheral neuropathy in ART-experienced patients: Prevalence and risk factors. J NeuroVirology. 19(6): 557-564. https://doi.org/10.1007/s13365-013-0216-4

Cysique, L.A.J., Maruff, P. and Brew, B.J. (2004) Antiretroviral therapy in HIV infection: Are neurologically active drugs important? Arch Neurol. 61(11):1699-1704. https://doi.org/10.1001/archneur. 61.11.1699

Das, K. and Arnold, E. (2013) HIV-1 reverse transcriptase and antiviral drug resistance. Part 1. Current Opinion Virology. 3(2):111-118. https://doi.org/10.1016/j.coviro.2013.03.012

Daverey, A. and Agrawal, S.K. (2016) Curcumin alleviates oxidative stress and mitochondrial dysfunction in astrocytes. Neurosci. 333:92-103. https://doi.org/10.1016/j.neuroscience.2016.07.012

De Clercq, E. (2009) Anti-HIV drugs: 25 compounds approved within 25 years after the discovery of HIV. Int J Antimicrob Agents. 33(4):307-320. https://doi.org/10.1016/j.ijantimicag.2008.10.010 de Oliveira, H.M., Damiani, A.P., Dias, R., Romão, P.R.T. and Andrade, V.M. (2014) Effect of antiretroviral drugs on the DNA damage in mice. Environ Toxicol Pharmacol. 37(1):390-395. https://doi.org/10.1016/j.etap.2013.12.011

Devine, M., Herrin, C., Warnack, W. and Dubey, D. (2018) Novel use of levodopa in human immunodeficiency virus encephalopathy-mediated parkinsonism in an adult. J Postgraduate Med. 64(1): 53-55. https://doi.org/10.4103/jpgm.JPGM $674 \quad 16$ Ellman, G.L. (1959) Tissue sulfhydryl groups. Arch Biochem Biophysics. 82(1): 70-77. https://doi.org /10.1016/0003-9861(59)90090-6

Ferrer, K. and Rakhmanina, N. (2013) Neuropsychiatric effects of tenofovir in comparison with other antiretroviral drugs. Neurobehavioral HIV Medicine, 5. https://doi.org/10.2147/NBHIV.S24888 Feuers, R.J., Weindruch, R., Leakey, J.E.A., Duffy, P. H. and Hart, R.W. (1997) Increased effective activity of rat liver catalase by dietary restriction. J Am Aging Association. 20(4):215-220. https://doi.org/10.1007/ s11357-997-0021-1

Fields, J.A., Swinton, M.K., Carson, A., Soontornniyomkij, B., Lindsay, C., Han, M.M., et al. (2019) Tenofovir disoproxil fumarate induces peripheral neuropathy and alters inflammation and mitochondrial biogenesis in the brains of mice. Sci Rep. 9:17158. doi:10.1038/s41598-019-53466-x

Funes, H.A., Blas-Garcia, A., Esplugues, J.V. and Apostolova, N. (2015) Efavirenz alters mitochondrial respiratory function in cultured neuron and glial cell lines. J Antimicrob Chemother. 70(8):2249-2254. https://doi.org/10.1093/jac/dkv098

Gabbai, A.A., Castelo, A. and Oliveira, A.S.B. (2013) HIV peripheral neuropathy. In: Handbook of Clinical Neurology. 115:515-529. https://doi.org/10.1016/ B978-0-444-52902-2.00029-1

Ganor, Y., Real, F., Sennepin, A., Dutertre, C.A., Prevedel, L., Xu, L., et al. (2019). HIV-1 reservoirs in urethral macrophages of patients under suppressive antiretroviral therapy. Nat Microbiol. 4(4):633-644. https://doi.org/10.1038/s41564-018-0335-z

Gelders, G., Baekelandt, V. and Van der Perren, A. (2018) Linking neuroinflammation and neurodegeneration in parkinson's disease. J Immunol Res. 2018: 4784268. https://doi.org/10.1155/2018 14784268

Grankvist, K., Marklund, S., Sehlin, J. and Taljedal, I. B. (1979) Superoxide dismutase, catalase and scavengers of hydroxyl radical protect against the toxic action of alloxan on pancreatic islet cells in vitro. Biochem. J. 182(1): 17-25. https://doi.org/10.1042/ bj1820017

Grant, I., Atkinson, J.H., Hesselink, J.R., Kennedy, C.J., Richman, D.D., Spector, S. A., et al. (1988) Human immunodeficiency virus-associated neurobehavioural disorder. J Royal College of Physicians London. 22(3): 149-157. http://www.ncbi. nlm.nih.gov/pubmed/3411542

Gray, L.R., Tachedjian, G., Ellett, A.M., Roche, M.J., Cheng, W.-J., Guillemin, G.J., et al. (2013) The NRTIs lamivudine, stavudine and zidovudine have reduced HIV-1 inhibitory activity in astrocytes. PLOS ONE. 8(4): e62196. https://doi.org/10.1371/ JOURNAL.PONE.0062196

Guillamón-Vivancos, T., Gómez-Pinedo, U. and Matías-Guiu, J. (2015) Astrocytes in neurodegenerative diseases (I): function and 
molecular description. Neurología (English Edition). 30(2):119-129. https://doi.org/10.1016/j.nrleng.2014. 12.005

Guo, C., Sun, L., Chen, X. and Zhang, D. (2013) Oxidative stress, mitochondrial damage and neurodegenerative diseases. Neural Regen Res. 8(21):2003-2014.

Heaton, R.K., Franklin, D.R., Deutsch, R., Letendre, S., Ellis, R.J., Casaletto, K., et al. (2015) Neurocognitive change in the era of HIV combination antiretroviral therapy: The longitudinal charter study. Clin Infectious Dis. 60(3):473-480. https://doi.org/ 10.1093/cid/ciu862

Hirrlinger, J, Schulz, J.B. and Dringen, R. (2002) Glutathione release from cultured brain cells: multidrug resistance protein 1 mediates the release of GSH from rat astroglial cells. J Neurosci Res. 69(3):318-326.

Hissin, P.J. and Hilf, R. (1976) A fluorometric method for determination of oxidized and reduced glutathione in tissues. Analytical Biochem. 74(1):214-226. https://doi.org/10.1016/0003-2697(76)90326-2

Hung, K.M., Chen, P.C., Hsieh, H.C. and Calkins, M. J. (2017) Mitochondrial defects arise from nucleoside/nucleotide reverse transcriptase inhibitors in neurons: Potential contribution to HIV-associated neurocognitive disorders. Biochimica et Biophysica Acta. 1863(2):406-413. https://doi.org/10.1016/i. bbadis.2016.11.017

Ikekpeazu, J.E., Orji, O.C., Uchendu, I.K. and Ezeanyika, L.U. (2020) Mitochondrial and oxidative impacts of short and long-term administration of HAART on HIV patients. Current Clin Pharmacol. 15(2):110-124.

Letendre, S., Marquie-Beck, J., Capparelli, E., Best, B., Clifford, D., Collier, A.C., et al. (2008). Validation of the CNS penetration-effectiveness rank for quantifying antiretroviral penetration into the central nervous system. Arch Neurol, 65(1):65-70. https://doi.org/10.1001/archneurol.2007.31

Llibre, J.M., Hung, C.C., Brinson, C., Castelli, F., Girard, P.M., Kahl, L.P., et al. (2018). Efficacy, safety, and tolerability of dolutegravir-rilpivirine for the maintenance of virological suppression in adults with HIV-1: phase 3, randomised, non-inferiority SWORD1 and SWORD-2 studies. Lancet. 391(10123): 839849. https://doi.org/10.1016/S0140-6736(17)33095-7 Lorber, M. (2013) A case of possible darunavir/ritonavir-induced peripheral neuropathy: case description and review of the literature. Journal of the International Association of Providers of AIDS Care. 12(3):162-165. https://doi.org/10.1177/ 2325957412471993

Lu, X., Ma, L., Ruan, L., Kong, Y., Mou, H., Zhang, Z., et al. (2010) Resveratrol differentially modulates inflammatory responses of microglia and astrocytes. J Neuroinflammation. 7(1): 1-14. https://doi.org/ 10.1186/1742-2094-7-46

Maga, G., Radi, M., Gerard, M.-A., Botta, M. and Ennifar, E. (2010) HIV-1 RT Inhibitors with a Novel
Mechanism of Action: NNRTIs that Compete with the Nucleotide Substrate. Viruses. 2(4):880-899. https://doi.org/10.3390/v2040880

Maioli, S., Båvner, A., Ali, Z., Heverin, M., Ismail, M.A.M., Puerta, E., et al. (2013) Is it possible to improve memory function by upregulation of the cholesterol 24S-hydroxylase (CYP46A1) in the brain? PLoS One 8: e68534-e68534

Medeiros, R. and LaFerla, F. M. (2013) Astrocytes: Conductors of the Alzheimer disease neuroinflammatory symphony. In Exp Neurol. 239:1):133-138. https://doi.org/10.1016/j.expneurol. 2012.10.007

Pejman, S., Kamarehei, M., Riazi, G., Pooyan, S. and Balalaie, S. (2020) Ac-SDKP ameliorates the progression of experimental autoimmune encephalomyelitis via inhibition of ER stress and oxidative stress in the hippocampus of C57BL/6 mice. Brain Res Bull. 154: 21-31. https://doi. org/10.1016/j.brainresbull.2019.09.014

Peter, A.I., Udoh, K.P. and Ekanem, A.U. (2017) Histomorphological effects of Lamivudine on the cerebellum of Wistar rats. Histol, Cytol Embryo., 1(3): $1-4$.

Petrov, A.M., Lam, M., Mast, N., Moon, J., Li, Y., Maxfield, E., et al. (2019). CYP46A1 Activation by efavirenz leads to behavioral improvement without significant changes in amyloid plaque load in the brain of 5XFAD mice. Neurotherapeutics. 16(3):710724. https://doi.org/10.1007/s13311-019-00737-0

Prevedel, L., Ruel, N., Castellano, P., Smith, C., Malik, S., Villeux, C., et al. (2019) Identification, Localization, and Quantification of HIV Reservoirs Using Microscopy. Current Protoc Cell Biol. 82(1). https://doi.org/10.1002/cpcb.64

Robertson, K., Liner, J. and Meeker, R.B. (2012) Antiretroviral neurotoxicity. J NeuroVirology. 18(5): 388-399. https://doi.org/10.1007/s13365-012-0120-3 Robertson, K.R., Smurzynski, M., Parsons, T.D., Wu, K., Bosch, R.J., Wu, J. et al. (2007) The prevalence and incidence of neurocognitive impairment in the HAART era. AIDS. 14:1915-1921. doi:10.1097/ QAD.0b013e32828e4e27

Rojo, L.E., Fernández, J.A., Maccioni, A.A., Jimenez, J.M. and Maccioni, R.B. (2008) Neuroinflammation: implications for the pathogenesis and molecular diagnosis of Alzheimer's disease. Archiv Med Res. 39(1):1-16. https://doi.org/10.1016/j.arcmed.2007.10. $\underline{001}$

Ru, W., and Tang, S. J. (2017) HIV-associated synaptic degeneration. Mol Brain. 10(1). https://doi. org/10.1186/s13041-017-0321-z

Saylor, D., Dickens, A.M., Sacktor, N., Haughey, N., Slusher, B., Pletnikov, M., et al. (2016) HIVassociated neurocognitive disorder - Pathogenesis and prospects for treatment. Nat Rev Neurol. 12(4):234-248. https://doi.org/10.1038/nrneurol.2016. $\underline{27}$

Schifitto, G., Yiannoutsos, C.T., Ernst, T., Navia, B. A., Nath, A., Sacktor, N. et al. (2009) Selegiline and 
oxidative stress in HIV-associated cognitive impairment. Neurol. 73(23):1975-1981. https://doi. org/10.1212/WNL.0b013e3181c51a48

Sen, S., Chakraborty, R., Sridhar, C., Reddy, Y.S.R. and De, B. (2010) Free radicals, antioxidants, diseases and phytomedicines: current status and future prospect. Int J Pharmaceut Sci Rev Res. 3(1). www.globalresearchonline.net

Shibata, N. and Kobayashi, M. (2008) The role for oxidative stress in neurodegenerative diseases. Brain Nerve. 60(2):157-170. https://doi.org/10.5607/en.201 $\underline{5.24 .4 .325}$

Smith, R.L., Tan, J.M.E., Jonker, M.J., Jongejan, A., Buissink, T., Veldhuijzen, S., et al. (2017) Beyond the polymerase-y theory: Production of ROS as a mode of NRTI-induced mitochondrial toxicity. PLoS ONE. 12(11): e0187424. https://doi.org/10.1371/journal.pon e.0187424

Soontornniyomkij, V., Umlauf, A., Soontornniyomkij, B., Gouaux, B., Ellis, R.J., Levine, A.J., et al. (2018) Association of antiretroviral therapy with brain aging changes among HIV-infected adults. AIDS. 32(14): 2005-2015. https://doi.org/10.1097/QAD.000000000 $\underline{0001927}$

Soriano, V., Fernandez-Montero, J.V., BenitezGutierrez, L., Mendoza, C.D., Arias, A., Barreiro, P., et al. (2017) Dual antiretroviral therapy for HIV infection. Expert Opinion on Drug Safety. 16(8):923932. https://doi.org/10.1080/14740338.2017.1343300 Tamagno, E., Guglielmotto, M., Aragno, M., Borghi, R., Autelli, R., Giliberto, L., et al. (2008) Oxidative stress activates a positive feedback between the $\mathrm{Y}$ and $\beta$-secretase cleavages of the $\beta$-amyloid precursor protein. J Neurochem. 104(3):683-695. https://doi.org/10.1111/j.1471-4159.2007.05072.x Tibalinda, P., Sempombe, J., Shedafa, R., Masota, N., Pius, D., Temu, M., et al. (2016) Formulation development and optimization of lamivudine $300 \mathrm{mg}$ and tenofovir disoproxil fumarate (TDF) $300 \mathrm{mg}$ FDC tablets by D-optimal mixture design. Heliyon. 2(12):e00207.

Vivithanaporn, P., Asahchop, E.L., Acharjee, S., Baker, G.B. and Power, C. (2016) HIV protease inhibitors disrupt astrocytic glutamate transporter function and neurobehavioral performance. AIDS. 30(4): 543-552. https://doi.org/10.1097/QAD.00000 $\underline{00000000955}$

WHO (2021) World Health Oganisation Fact Sheets Detail HIVIAIDS. Newsroom. 14-07-2021. https://www.who.int/news-room/fact-sheets/detail/hivaids

$\overline{X u}$, P., Wang, Y., Qin, Z., Qiu, L., Zhang, M., Huang, Y., et al. (2017). Combined medication of antiretroviral drugs tenofovir disoproxil fumarate, emtricitabine, and raltegravir reduces neural progenitor cell proliferation in vivo and in vitro. $\mathrm{J}$ Neuroimmune Pharmacol. 12(4):682-692. https://doi. org/10.1007/s11481-017-9755-4

Zulu, S.S., Simola, N., Mabandla, M.V. and Daniels, W.M.U. (2018) Effect of long-term administration of antiretroviral drugs (tenofovir and nevirapine) on neuroinflammation and neuroplasticity in mouse hippocampi. J Chem Neuroanat. 94:86-92. doi:10.10 16/j.jchemneu.2018.10.003

Cite as Dosumu, O., Akang, E., Edem, E., Afolayan, S. and Akanmu, S.A. (2021) Neurocognitive and neuroarchitectural changes in the prefrontal cortex of Wistar rats treated with highly active antiretroviral therapy. Nig. J. Neurosci. 12(2):67-75. http://doi.org/10.47081/njn2021.12.2/004

(c) Copyright Nigerian Journal of Neuroscience. All rights reserved. 